\title{
Complexation and Structure Elucidation of the Axial Conformers of Mono- and ( \pm )-trans-1,2-Disubstituted Cyclohexanes by Enantiopure Alleno-Acetylenic Cage Receptors
}

\author{
Cornelius Gropp ${ }^{\S}$ and Nils Trapp \\ §SCS-DSM Award for best poster presentation in Organic Chemistry
}

\begin{abstract}
Single crystal X-ray diffraction is a powerful method to unambiguously characterize the structure of molecules with atomic resolution. Herein, we review the molecular recognition of the (di)axial conformers of mono- and ( \pm -trans-1,2-disubstituted cyclohexanes by enantiopure alleno-acetylenic cage receptors in solution and in the solid state. Single crystals of the host-guest complexes suitable for X-ray diffraction allow for the first time to study the dihedral angles of a series of mono- and ( \pm -trans-1,2-disubstituted cyclohexanes in their (di)axial chair conformation. Theoretical studies indicate negligible influence of the host structure on the guest conformation, suggesting that the structural information obtained from the host-guest complexes give insight into the innate structures of mono- and ( \pm -trans-1,2-disubstituted cyclohexanes. Strong deviation of the dihedral angles $\left.\theta_{\mathrm{a}, \mathrm{a}} \mathrm{X}-\mathrm{C}(1)-\mathrm{C}(2)-\mathrm{X}\right)$ from the idealized $180^{\circ}$ are observed, accompanied by substantial flattening of the ring dihedral angles $\rho(X-C(1)-C(2)-C(3))$.
\end{abstract}

Keywords: Alleno-acetylenes $\cdot$ Cage compounds $\cdot$ Computational chemistry $\cdot$ Molecular recognition $\cdot \mathrm{X}$-ray diffraction

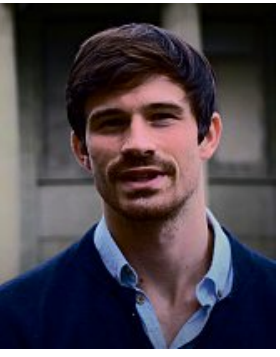

Cornelius Gropp conducted a oneyear civil service in Kundapura, India, before starting his BSc at the University of Heidelberg in 2009. After a one-year exchange at the École Polytechnique in Palaiseau he received his BSc in 2012. Subsequent to a visiting research stay

\footnotetext{
${ }^{*}$ Correspondence: C. Gropp
}

in the group of Prof. P. S. Baran at the Scripps Research Institute in La Jolla, USA, he moved to ETH Zurich to obtain his MSc degree under the guidance of Prof. E. M. Carreira and Prof. F. Diederich in 2014. After an internship at the Novartis Institute for Biomedical Research (NIBR/ GDC) he joined the research group of Prof. F. Diederich for his PhD studies at ETH Zurich in 2014. He has been supported by the Studienstiftung des Deutschen Volkes during his BSc, MSc and PhD studies.

\section{Introduction}

Structure elucidation is the process of determining the chemical structure of a compound. ${ }^{[1]}$ Despite the constantly evolving techniques to characterize the structure of molecules, single crystal X-ray diffraction remains the most important method to provide accurate structural information with atomic resolution. ${ }^{[2]}$ Most molecules and molecular complexes can in principle be crystallized, but the disorder in their solid-state assemblies often prevents the determination of high-resolution structures. ${ }^{[2 b, e, 3]}$ Early techniques, such as clathrate-type inclusion complexation and crystallization in porous complexes, were developed in an attempt to overcome these challenges by integrating guest molecules into an inorganic or organic host lattice. ${ }^{[4]}$ Through inclusion into host lattices, the guest molecules interact with the host, which can result in an ordering of the molecules and a lowering of their motional degrees of freedom. Thereby, the orientational disorder can be effectively decreased, allowing for high-resolution structure determination. Few of these systems, however, showed general applicability for the structure determination of molecules, mainly due to the strong influence of the size and shape of the guest on the crystal formation. ${ }^{[4 a]}$ More recently, coordination of small molecules into crystalline metal-organic frameworks (MOFs) ${ }^{[5]}$ and their soaking into crystalline sponges ${ }^{[6]}$ emerged as alternatives. The main difference to the clathrate-type inclusion is that the single crystal is formed prior to guest complexation. Suitable single crystals are subsequently soaked with the guests or the guest is coordinatively aligned within the framework of the host structure. ${ }^{[5,6]}$ The porous nature of the metal-organic frameworks results in large and open voids capable of incorporating a large variety of molecules. The drawback of the porous nature of the MOFs is that it often results in 
lower occupancies of the guest molecules, which can lead to lower resolution of the guest structures. ${ }^{[5-7]}$ In contrast, molecular receptors with confined cavities can form multiple interactions with guest molecules. With a suitable crystallization protocol, guest complexation can lead to the formation of single crystals suitable for X-ray diffraction. While molecular recognition of the guest with the host often leads to higher guest occupancies and lower disorder, the specificity of the receptor can limit the choice of suitable guest molecules. ${ }^{[7,8]}$

Here we review a strategy for the complexation and co-crystallization of cyclohexane and its mono- and ( \pm )-trans-1,2disubstituted derivatives in the interior of enantiopure cage receptors. ${ }^{[9]}$ Solution binding studies give insight into the affinity of the guest molecules towards the receptors and, for the stronger binding guests, enables the study of the host-guest complexes. Molecular structures obtained from single crystal X-ray diffraction allow the characterization of the molecular structures of the encapsulated guests with atomic resolution. Our results show that the guest molecules are complexed in their axial/diaxial chair conformations with dihedral angles $\theta_{\text {aa }}(\mathrm{X}-\mathrm{C}(1)-\mathrm{C}(2)-\mathrm{X})$ deviating substantially from the idealized angle of $180^{\circ} .{ }^{[9 \mathrm{~b}]}$ We investigated the structures of the host-guest complexes and of the isolated guest molecules with quantum chemical methods and find that the encapsulation hardly affects the structure of the guest molecules. ${ }^{[9 b]}$ The results regarding the structure of the guest molecules obtained from the experimental investigation of the host-guest complexes may, hence, be transferred to the isolated guest structures, which further highlights the value of this host as a means to elucidate the structure of cyclohexane derivatives.

\section{The Host: Enantiopure Alleno- Acetylenic Cage Receptors}

Enantiopure alleno-acetylenic cage (AAC) receptors are constructed from a methylene-bridged resorcin[4]arene core to which four homochiral alleno-acetylenes with $\mathrm{OH}$ termini are attached, giving access to $(P)_{4}$ - and $(M)_{4}$-configured AACs (Fig. 1, only $(P)_{4}$-configured receptor is shown). ${ }^{[9 a]}$

The AAC receptors undergo solventdependent binary conformational switching between a closed cage conformation and an open state by rotation around a $\mathrm{C}-\mathrm{C}$ bond (Fig. 1, left). Both states were characterized in solution by NMR and IR spectroscopic studies and by single crystal $\mathrm{X}$-ray diffraction in the solid state. ${ }^{[9 \mathrm{a}]}$ In the closed cage conformation (Fig. 1), the $\mathrm{OH}$ termini of the alleno-acetylenic arms form

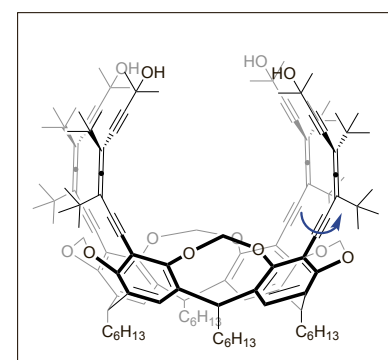

$(P)_{4}-\mathrm{AAC}$

Lewis structure

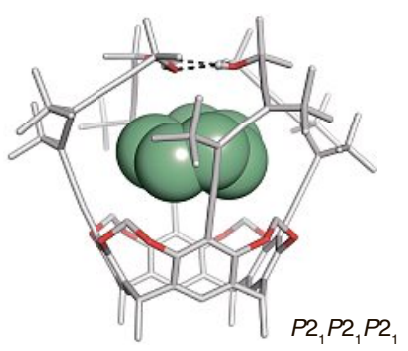

$(P)_{4}$ - AAC $\supset$ cycloheptane $\mathrm{X}$-ray crystal structure

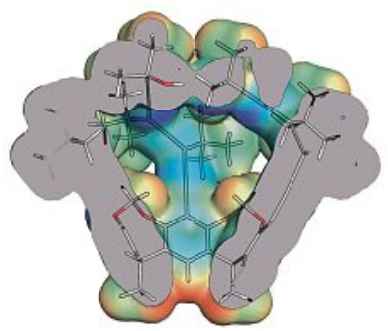

$(P)_{4}-\mathrm{AAC}$

electrostatic potential map

Fig. 1. Lewis structure of $(P)_{4}-\mathrm{AAC}$ (left), blue arrow indicates conformational switching towards the open state; X-ray co-crystal structure of $(P)_{4}$-AAC $\supset$ cycloheptane, hydrogens and $n$-hexyl chains omitted for clarity, complexed guest depicted in sphere representation (center); electrostatic potential map of $(P)_{4}-\mathrm{AAC}$ based on the data calculated in ref. [9b] (right); Only $(P)_{4}^{-}$ configured structures depicted. ${ }^{[9]}$

a cyclic four-fold hydrogen-bonding array, which creates a highly confined cavity. This binary conformational switching is accompanied by strong changes in the associated electronic circular dichroism (ECD) spectra, allowing for a spectroscopic readout of the conformational changes. The combination of an interior capable of guest complexation together with the highly sensitive optical readout through ECD enables to identify and quantify guest binding. [9a] The interior of the host can be characterized by means of an electrostatic potential map. While the outer surface of the receptor is largely characterized by a neutral electrostatic potential (Fig. 1, right), the interior of the host displays areas of negative electrostatic potential associated with the oxygen atoms closing the cage at the top, and with the aromatic and acetylenic moieties within the host. This enables first insights into which guests could interact favorably with the interior of the host and how complementarity of the host-guest interaction could lead to selective binding. [9b]

\section{The Guests: Complexation and Structural Elucidation of Mono- and ( \pm )-trans-1,2-Disubstituted Cyclohexanes}

The isolation and characterization of single conformers of mono- and ( \pm )-trans-1,2-disubstituted cyclohexanes is difficult due to the rapid isomerization process between their (di)equatorial and (di)axial conformers. ${ }^{[10]}$ The existence of both conformers together with the respective preference for either conformation has previously been studied in solution by IR and at low temperature by NMR spectroscopies, and enabled the quantitative determination of their conformational energies $\left(A\right.$-values). ${ }^{[10 b, 11]}$ While the equatorial conformations are generally preferred for monosubstituted cyclohexanes (positive $A$-values), the diaxial conformation be- comes the more stable conformation for ( \pm )-trans-1,2-dihalocyclohexanes (negative $A$-values). ${ }^{[10,12]}$ Despite continuous research on substituted cyclohexanes, no conformational isomer of the mono- or ( \pm )-trans-1,2-disubstituted cyclohexanes has ever been isolated at ambient temperatures in solution, and only few X-ray cocrystal structures have been reported. [10b,13] In this context, we sought to utilize AACs as receptors to trap the single conformers of monosubstituted cyclohexanes (methyl-, fluoro-, chloro-, bromo- and iodocyclohexane) and selected ( \pm )-trans-1,2-disubstituted analogs ( \pm -trans-1,2-dichloro-, -dibromo- and -dimethylcyclohexane) in solution and in the solid state.

We developed a general protocol to obtain single crystals of the solid-state inclusion complexes suitable for X-ray diffraction. $\left.{ }^{9 b}\right]$ The molecular structures obtained from X-ray diffraction show the exclusive complexation of the axial/diaxial conformers of this series of mono- and ( \pm )-trans-1,2-disubstituted cyclohexanes with the single exception of methylcyclohexane. ${ }^{[9]}$ In the latter, the guest binds in the equatorial conformation with one methyl group of the host rotated into the interior of the cavity, compensating for the smaller size of the guest.[9a] The molecular structures determined by X-ray diffraction enabled us to analyze the diaxial dihedral angles of this series in detail. The dihedral angle $\theta(\mathrm{X}-\mathrm{C}(1)-$ $\mathrm{C}(2)-\mathrm{H})$ decreases from $-173^{\circ}$ for fluorocyclohexane to $-165^{\circ}$ for chloro-, bromo- and iodocyclohexane (Fig. 2). ${ }^{[9 b]}$ This decrease in the dihedral angle is accompanied by a flattening of the ring dihedral angles $\rho(\mathrm{X}-\mathrm{C}(1)-\mathrm{C}(2)-\mathrm{C}(3))$ from $+53^{\circ}$ for fluorocyclohexane to $+73^{\circ}$ for iodocyclohexane (Fig. 2A). ${ }^{[9 \mathrm{~b}]}$ In the series of ( \pm )-trans-1,2-disubstituted cyclohexanes, the dihedral angles $\theta_{\mathrm{a}, \mathrm{a}}(\mathrm{X}-\mathrm{C}(1)-\mathrm{C}(2)-\mathrm{X})$ further decrease down to $-147^{\circ}$ for $(R, R)$-trans-1,2-bromofluorocyclohexane and to $-146^{\circ}$ for $(R, R)$-trans-1,2-dime- 
thylcyclohexane complexed to $(P)_{4}$-AAC. Again, this decrease in the dihedral angle $\theta_{\mathrm{a}, \mathrm{a}}$ is accompanied by a flattening of the ring dihedral angles $\rho(\mathrm{X}-\mathrm{C}(1)-$ $\mathrm{C}(2)-\mathrm{C}(3))$ of up to $+79^{\circ}$ for $(R, R)$-trans1,2-bromofluorocyclohexane (Fig. 2B). [9b]

The strong deviation from the idealized dihedral angle $\theta_{\text {a.a }}$ of $180^{\circ}$ for all investigated cyclohexane derivatives raised the question of how much the encapsulating host affects the structures of the guest molecules, and hence $\theta_{\mathrm{a}, \mathrm{a}}$. The crystallographic data already suggest that this effect is likely to be small, because the observed host-guest contacts exceed the sum of their respective covalent van der Waals radii (heavy atom distances $\geq 3.50 \AA$ ). ${ }^{[9 b]}$ We characterized the interactions between $( \pm)$-trans-1,2-dimethylcyclohexane and $(P)_{4}$-AAC, and between methylcyclohexane and $(P)_{4}$-AAC with the non-covalent interaction measures introduced in ref. [14] (see Fig. 3 for a graphical representation). Fig. 3 shows that methylcyclohexane and ( \pm )-trans-1,2dimethylcyclohexane exhibit allover enveloping dispersive interactions without strong (repulsive or attractive) interactions with the host. The absence of strong interactions suggests that the trapped guest structures might not be strongly affected by the host. This can be directly probed by quantum chemical optimization and comparison of the isolated and encapsulated guest structures. In ref. [9b], we found that the root-mean-square deviations of the atomic positions in the isolated and encapsulated guest structures do not exceed $0.02 \AA$, i.e. the trapped guest structures closely resemble the isolated ones. Consequently, the differences of the dihedral angles $\theta_{a, a}$ in the isolated and complexated guest structures are small (on average $1^{\circ}$, Fig. 4). A direct comparison of the measured and calculated $\theta_{\mathrm{a}, \mathrm{a}}$ in the host-guest complexes shows a satisfactory agreement for some structures $(( \pm)$-trans-1,2-dihalocyclohexanes, Fig. 4), but large deviations of up to $15^{\circ}$ for others $(( \pm)$-trans-1,2-dimethylcyclohexane and monohalocyclohexanes, Fig. 4). While this disagreement seems large, it can be traced back to the flexibility of the cyclohexane scaffold. A reduction of $\theta_{\mathrm{a}, \mathrm{a}}$ in $(P)_{4}-\mathrm{AAC} \supset( \pm)$-trans1,2-dimethylcyclohexane by up to $15^{\circ}$ requires less than $1 \mathrm{kcal} \mathrm{mol}^{-1} \cdot{ }^{[9 \mathrm{~b}]}$ Our results indicate that the reason for $\theta_{\text {a }}$ to be significantly smaller than the idealized angle of $180^{\circ}$ is not the encapsulation, because the dihedral angles $\theta_{\mathrm{a}, \mathrm{a}}$ are also smaller than $180^{\circ}$ in the isolated cyclohexane derivatives.

Following the analysis of the molecular structures obtained by X-ray diffraction, we conducted solution complexation studies by ${ }^{1} \mathrm{H}$ NMR and ECD spectroscopic titrations in a non-competitive solvent $\left(d_{18}\right.$-n-octane and $n$-octane $)$ at $293 \mathrm{~K} \cdot{ }^{[9 a}$

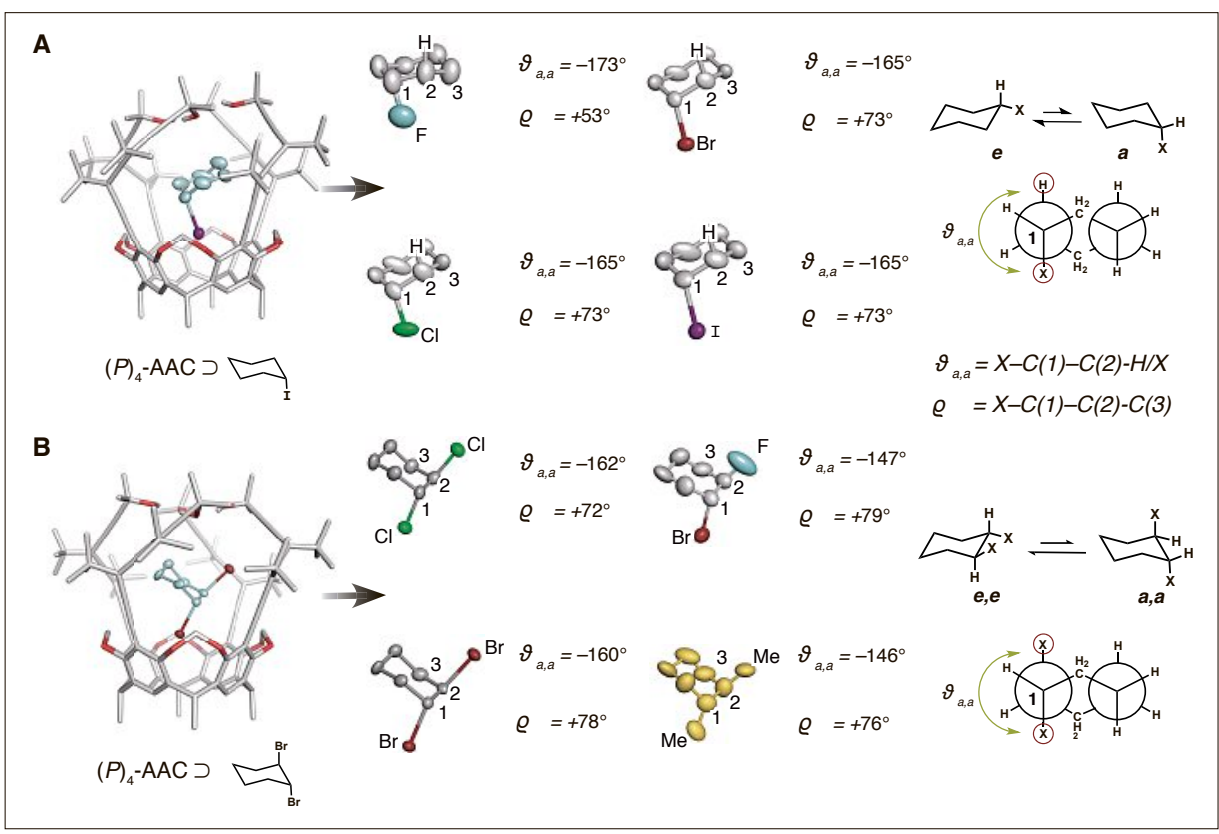

Fig. 2. (A) X-ray co-crystal structures of monohalocyclohexanes obtained from $(P)_{4}-\mathrm{AAC}$ in the axial conformation with fluoro-, chloro-, bromo- and iodocyclohexane; (B) X-ray co-crystal structures of $( \pm)$-trans-1,2-disubstituted cyclohexanes in the diaxial conformation obtained from $(P)_{4}$-AAC with ( \pm )-trans-1,2-dichloro-, -dibromo-, -bromofluoro- and -dimethylcyclohexane; only $(R, R)$-configured guests are shown; dihedral angles of the complexed guests in their (di)axial chair conformation are given in ${ }^{\circ} \cdot{ }^{[9]}$

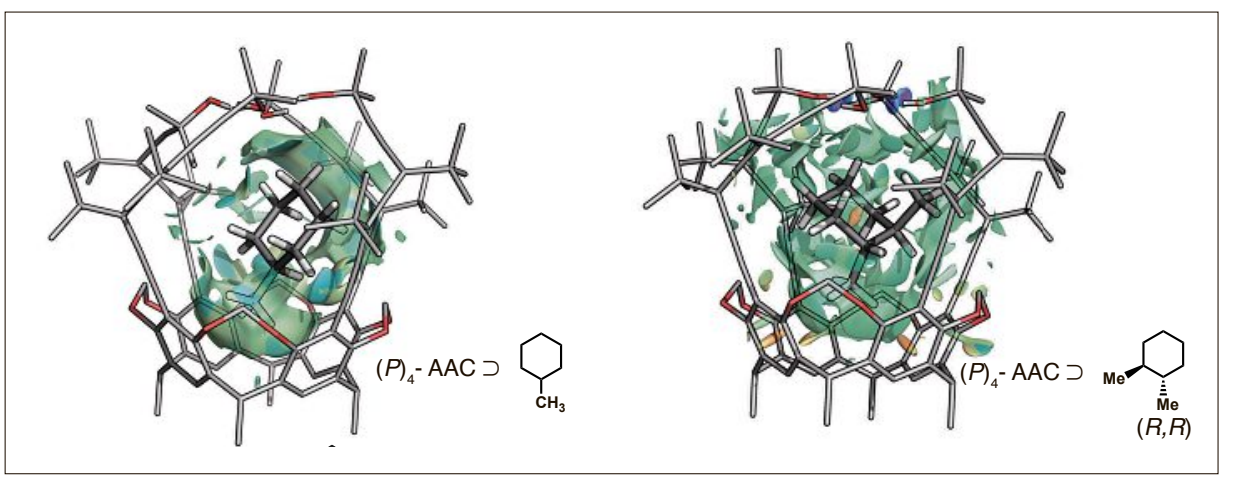

Fig. 3. Isosurface of the reduced density gradient $s(r)$ of density functional theory for $s(r)=0.55$ revealing the interaction between $(R, R)$-trans-1,2-dimethylcyclohexane and $(P)_{4}-\mathrm{AAC}$ (left) and between methylcyclohexane and $(P)_{4}$-AAC (right) within a radius of $4.5 \AA$ around the centroid of the guest molecule based on the data calculated in ref. [9b]. The surfaces are colored on a blue-green-red scale according to the type of interactions with which they are associated: Blue indicates strongly attractive interactions such as hydrogen-bonding, and green indicates dispersive interactions; red would indicate steric clashes, but no such red regions emerged in the analysis. We refer to ref. [14] for a detailed discussion on how the types of interaction are assigned. Element color code: carbon in host, light gray; carbon in guest, dark gray; oxygen, red; hydrogen, white. Hydrogen atoms of the host are omitted for clarity.

The binding studies quantify the ability of the AACs to complex mono- and ( \pm )-trans-1,2-disubstituted cyclohexanes also in solution. ${ }^{[9 b]}$ Slow exchange of the stronger binding guests on the NMR timescale, such as the iodo- (at $293 \mathrm{~K}$ ), dichloro- (at $273 \mathrm{~K}$ ), and dibromo- (at $293 \mathrm{~K}$ ) derivatives, enabled the characterization of the inclusion complexes in solution, further substantiating their exclusive binding in the axial/diaxial conformation. ${ }^{[9 b]}$ The nature of the substituent together with the substituent-dependent preference for the (di)equatorial or (di) axial conformation strongly affects the association constant of the guest towards the receptor. In general, cyclohexane derivatives with large positive $A$-values show weaker binding, compared to derivatives with negative $A$-values. The binding affinities for monosubstituted cyclohexanes increase with the substituent in the order of $\mathrm{Me}<\mathrm{F}<\mathrm{Cl}<\mathrm{Br}<\mathrm{I}$. The substantial increase in the binding affinities of 270 $\mathrm{M}^{-1}$ for fluorocyclohexane to $18000 \mathrm{M}^{-1}$ for iodocyclohexane can be rationalized with the favorable halogen-bonding interactions of the iodocyclohexane with the 


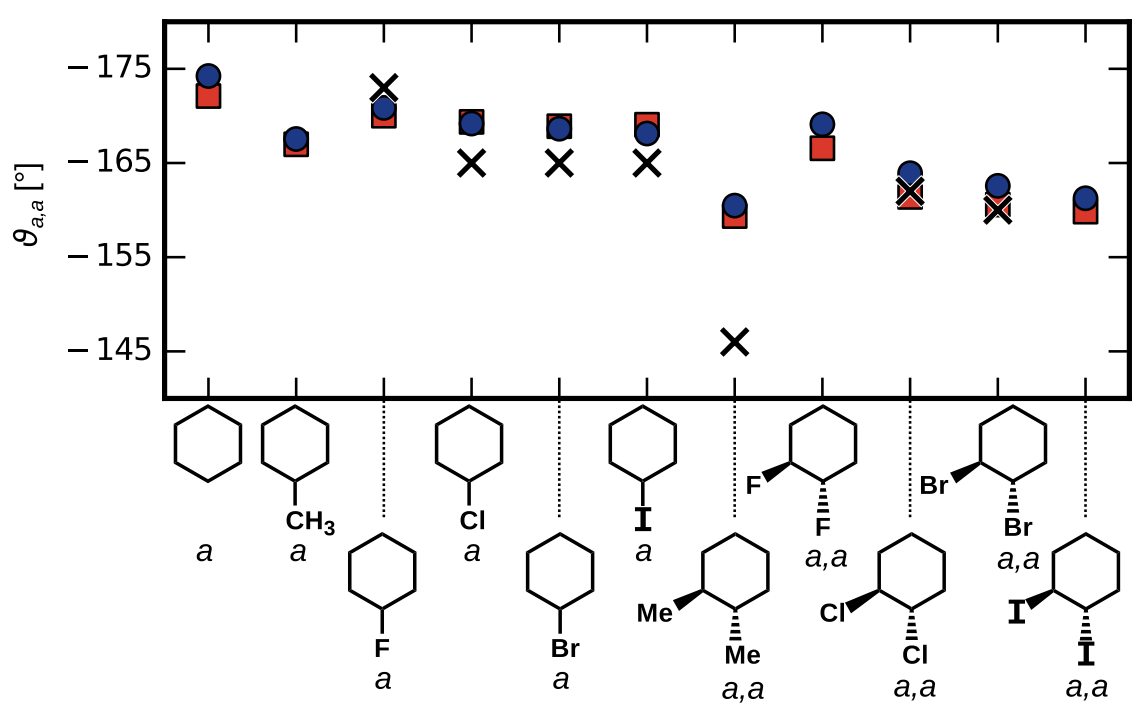

Fig. 4. Calculated dihedral angles $\theta_{\text {a }}$ in the isolated cyclohexane derivatives (blue circles) or in the host-guest complexes (red squares) in their axial (a) conformation reproduced from data presented in ref. [9b] in comparison to available experimental values (black crosses).

aromatic moieties of the resorcin[4]arene receptor. ${ }^{[9 b]}$ This halogen- $\pi$-interaction contributes to an increase in binding affinity of $\Delta \Delta G=-2.4 \mathrm{kcal} \mathrm{mol}^{-1}$ (both molecules show comparable $A$-values of -0.3 kcal $\left.\mathrm{mol}^{-1}\right) \cdot{ }^{[9 \mathrm{~b}]}$ The contribution of both the conformational energies and favorable halogen-bonding interactions to the binding affinity is observed in the series of ( \pm )-trans-1,2-disubstituted cyclohexanes: The association constant increases upon halogen addition from $110 \mathrm{M}^{-1}$ for $( \pm)$-trans-1,2-dimethylcyclohexane up to $29000 \mathrm{M}^{-1}$ for $( \pm)$-trans-1,2-dibromocyclohexane, ${ }^{[9 b]}$ with $( \pm)$-trans-1,2-dimethylcyclohexane favoring the diequatorial conformation $\left(A\right.$-value of $\left.+3.0 \mathrm{kcal} \mathrm{mol}^{-1}\right)$ and $( \pm)$-trans-1,2-dibromocyclohexane favoring the diaxial conformation ( $A$-value of $\left.-0.5 \mathrm{kcal} \mathrm{mol}^{-1}\right)$. The difference in conformational energy, together with the favorable halogen bonding interactions of the bromo-substituents with the $\pi$-system of the receptor, translates into the large increase in binding affinity of $\Delta \Delta G=-3.3$ kcal $\mathrm{mol}^{-1}$.

\section{Conclusion}

In summary, we reviewed the molecular recognition of a series of mono- and ( \pm )-trans-1,2-disubstituted cyclohexanes by alleno-acetylenic cage receptors. Molecular structures obtained from single crystal X-ray diffraction reveal the exclusive complexation of the (di) axial conformations of monohalo- and ( \pm -trans-1,2-disubstituted cyclohexanes, allowing for the first time to analyze a series of dihedral angles of monohalo- and
( \pm )-trans-1,2-disubstituted cyclohexanes in their (di)axial chair conformation. The dihedral angles $\theta_{\mathrm{a}, \mathrm{a}}(\mathrm{X}-\mathrm{C}(1)-\mathrm{C}(2)-$ $\mathrm{H} / \mathrm{X}$ ) show strong deviation from $180^{\circ}$ down to $-146^{\circ}$ for $(R, R)-( \pm)$-trans-1,2dimethylcyclohexane and $-147^{\circ}$ for $(R, R)$ ( \pm )-trans-1,2-bromofluorocyclohexane. Theoretical analysis of the isolated guest molecules shows close agreement with the complexed guest structures, demonstrating negligible influence of the host on the structure of the guest molecules. This further validates the utility of the AACs to capture single conformers of derivatives of cyclohexane for their structural elucidation. Slow exchange of guest molecules on the NMR timescale, associated with their stronger binding, allowed the characterization of the inclusion complexes in solution, further substantiating their exclusive binding in the axial/diaxial conformation. Solution binding studies give insight into the affinities of the guests depending on the nature of the substituent and the conformational energies associated with accessing the (di)axial conformer necessary for binding to the interior of the host. The difference in conformational energy, together with the favorable halogen bonding interactions, resulted in large increase in binding affinities of up to $\Delta \Delta G=-3.3 \mathrm{kcal}$ $\mathrm{mol}^{-1}$ for $( \pm)$-trans-1,2-dimethylcyclohexane compared to $( \pm)$-trans-1,2-dibromocyclohexane.

\section{Acknowledgements}

This study was supported by the Swiss National Science Foundation (SNF 200020_159802). C. G. was supported by the Studienstiftung des Deutschen Volkes. We thank T. Husch and M. Reiher for help in pre- paring the figures and for valuable comments on the manuscript. The research program on alleno-acetylenes was initiated by F. Diederich in the late 1990s and expanded into supramolecular chemistry in recent years. We are grateful for his support and encouragement.

Received: January 29, 2018

[1] a) 'Structure Elucidation in Organic Chemistry: The Search for the Right Tools', Eds. M-M. Cid, J. Bravo, Wiley-VCH, Weinheim, 2015.

[2] a) H.-B. Bürgi, J. D. Dunitz, Helv. Chim. Acta 1993, 76, 1115; b) J. D. Dunitz, 'X-ray Analysis and the Structure of Organic Molecules', Cornell University Press, Ithaca, 1995; c) J. D. Dunitz, A. Gavezzotti, Angew. Chem. Int. Ed. 2005, 44, 1766; d) G. M. Sheldrick, Acta Cryst. A 2008, 64, 112; e) L.-1. Ooi, 'Principles of X-ray Crystallography', Oxford University Press, Oxford, 2010; f) F. Diederich, N. Trapp, M. Wörle, Isr. J. Chem. 2017, 57, 39.

[3] A. Holden, P. Morrison, 'Crystals and Crystal Growing', MIT Press, Cambridge - Mass., 1982.

[4] a) 'Inclusion Compounds', Eds. J. L. Atwood; J. E. D. Davies, D. D. MacNicol, Academic Press, London, Vol. 1-2, 1984; b) F. Toda, Pure Appl. Chem. 2001, 73, 1137.

[5] S. Lee, E. A. Kapustin, O. M. Yaghi, Science 2016, 353, 808 .

[6] a) Y. Inokuma, S. Yoshioka, J. Ariyoshi, T. Arai, Y. Hitora, K. Takada, S. Matsunaga, K. Rissanen, M. Fujita, Nature 2013, 495, 461; b) M. Hoshino, A. Khutia, H. Xing, Y. Inokuma, M. Fujita, IUCrJ 2016, 3, 139.

[7] K. Rissanen, Chem. Soc. Rev. 2017, 46, 2638.

[8] Y.-M. Legrand, A. van der Lee, M. Barboiu, Science 2010, 329, 299.

[9] a) C. Gropp, N. Trapp, F. Diederich, Angew. Chem. Int. Ed. 2016, 55, 14444; b) C. Gropp, T. Husch, N. Trapp, M. Reiher, F. Diederich, $J$. Am. Chem. Soc. 2017, 139, 12190.

[10] a) F. R. Jensen, C. H. Bushweller, 'Conformational Preferences in Cyclohexanes and Cyclohexenes', in 'Advances in Alicyclic Chemistry', Eds. H. Harold; G. J. Karabatsos, Academic Press Inc., London, 1971, pp 139 194. b) S. H. Wilen, L. N. Mander, E. L. Eliel, 'Stereochemistry of Organic Compounds', John Wiley \& Sons, New York, 1994.

[11] a) P. Klaeboe, J. J. Lothe, K. Lunde, Acta Chem. Scand. 1956, 10, 1465; b) L. W. Reeves, K. O. Strømme, Can. J. Chem. 1960, 38, 1241.

[12] a) D. H. R. Barton, Experientia 1950, 6, 316; b) E. L. Eliel, Angew. Chem. Int. Ed. 1965, 4, 761.

[13] a) M. Kato, K. Tanaka, F. Toda, Supramol. Chem. 2001, 13, 175; b) L. Yeo, K. D. M. Harris, B. M. Kariuki, J. Solid State Chem. 2001, 156, 16; c) S. Hirano, S. Toyota, F. Toda, Chem. Commun. 2004, 2354; d) S. Hirano, S. Toyota, M. Kato, F. Toda, Chem. Commun. 2005, 3646; e) R. Bream, D. Watkin, A. Cowley, Acta Crystallogr. E 2006, 62, O785; f) K. Tanaka, S.-i. Wada, M. R. Caira, Tetrahedron 2007, 63, 9213; g) B. A. Palmer, B. M. Kariuki, A. Morte-Ródenas, K. D. M. Harris, Cryst. Growth Des. 2012, 12, 577.

[14] E. R. Johnson, S. Keinan, P. Mori-Sanchéz, J. Contreras-García, A. J. Cohen, W. Yang, J. Am. Chem. Soc. 2010, 132, 6498. 